\title{
Subacute massive pulmonary embolism
}

\author{
RJC HALL, * D MCHAFFIE, $†$ C PUSEY $\ddagger$ GC SUTTON $\$$
}

From the Cardiac Department, Brompton Hospital, London

SUMMARY Twenty-four patients with subacute massive pulmonary embolism were studied both during their initial illness and up to nine years after it. The most common mode of presentation was progressive dyspnoea over a two to 12 week period, which in some, but not all, patients was accompanied by pleuritic chest pain and haemoptysis. Physical signs at diagnosis usually suggested right heart strain and ventilation/perfusion mismatch and in the five patients with the highest pulmonary artery pressures the pulmonary component of the second sound was accentuated. The chest $x$-ray and electrocardiogram provided useful diagnostic information in most patients though occasionally they were normal. Early response to thrombolytic treatment was poor when compared with patients with acute pulmonary embolism but was occasionally dramatically successful, and heparin alone provided satisfactory treatment in the eight patients receiving it. Pulmonary embolectomy provided poor results and four of the five patients undergoing this form of treatment died. Nine patients died during the initial illness and in seven death was directly related to embolic disease. One patient died from neoplastic disease during follow-up. Though the prolonged illness, poor initial response to treatment, and absence of predisposing factors suggest that recurrent embolic aisease and late pulmonary hypertension might occur there was no evidence of this during a follow-up period of one to nine years (median five years).

Pulmonary embolic disease can present in many ways ranging from mild pleuritic pain to sudden fatal collapse. Since the presentation is so varied, classification of these patients into different clinical subgroups using the history and pulmonary angiographic findings is needed before possible differences in clinical course, response to treatment, and late prognosis can be considered.

This study was undertaken to extend our previous observations of one particular subgroup, subacute massive pulmonary embolism. We have previously defined these patients as having long histories (between two and 12 weeks) without a definite episode of cardiovascular collapse and pulmonary angiograms showing massive pulmonary embolism ( $>50 \%$ obstruction of major pulmonary arteries). This group of patients is of particular interest for two reasons.

(1) Since presentation is much less dramatic

\footnotetext{
*Present address: Royal Victoria Infirmary, Newcastle Upon Tyne. tPresent address: Wellington Hospital, Wellington 2, New Zealand. $¥$ Present address: Royal Postgraduate Medical School, London. §Present address: Hillingdon Hospital, Hillingdon, Middlesex.

Received for publication 6 August 1980
}

than in acute massive pulmonary embolism the diagnosis is often difficult to make. This study provides a detailed clinical description of this condition so that it can be recognised more readily.

(2) Since these patients have long histories, often lack a well-defined predisposing cause for embolic disease, and frequently show little angiographic improvement after initial treatment, they might be expected to be at particular risk from recurrent embolism and the development of thromboembolic pulmonary hypertension. This study, therefore, investigated the long-term prognosis of these patients with particular reference to these points.

\section{Patients and diagnosis}

The series consists of 24 consecutive patients (12 male and 12 female, age range 20 to 71 years) referred to the Brompton Hospital with subacute massive pulmonary embolism. Thirteen of these patients have been reported previously ${ }^{1}$; further information including up-to-date follow-up was obtained on these patients and 11 further patients added. 
DEFINITION OF SUBACUTE MASSIVE

PULMONARY EMBOLISM

The criteria used to define the group of patients were the same as previously reported. ${ }^{1}$ All patients had long histories (more than two weeks) without an episode of cardiovascular collapse, but had massive pulmonary embolism with more than 50 per cent obstruction of major pulmonary arteries at angiography or embolectomy. Pulmonary angiograms were scored 23 from 0 to 34 depending on the severity of pulmonary artery obstruction. A score of greater than 17 represented massive embolism.

All patients were seen during their initial illness by either RH or GCS. The diagnosis was confirmed by pulmonary angiography in 22 of the 24 patients and in the remaining two patients, early in the series, at pulmonary embolectomy.

METHOD OF FOLLOW-UP

Patients were recalled and examined by two of us (RH and DMcH). Particular notice was taken of symptoms and signs that might suggest recurrent pulmonary emboli or pulmonary hypertension. All patients had chest $x$-rays and 12 lead electrocardiograms. Right heart catheterisation, pulmonary angiography, and perfusion lung scans (using 99m technetium labelled albumin macroaggregates) were performed at follow-up in most, but not all, patients.

\section{Clinical presentation}

\section{HISTORY}

All the patients, by definition, had long histories without an episode of cardiovascular collapse precipitating their presentation. Increasing dyspnoea, either at rest or on exertion, was the most prominent symptom in all but one patient in whom mild dyspnoea was overshadowed by pleuritic pain and haemoptysis. Pleuritic pain occurred at some stage of the illness in 16 of the 24 patients and was accompanied by haemoptysis in six. Another six patients had haemoptysis without pleuritic pain, and in six patients persistent cough was also prominent. Two patients had dyspnoea as their only complaint.

Although, by definition, no patient presented with an acute episode of collapse three patients had a sudden acute exacerbation of symptoms during the course of their illness, which suggested that an acute embolic event had occurred at that time.

PREDISPOSING FACTORS

Eight patients had recognisable factors predisposing to thromboembolic disease. These were the contraceptive pill in four, pregnancy in one, primary lymphoedema and gross obesity in one, chronic lymphatic leukaemia in one, and systemic lupus erythematosis and a previous splenectomy in one. In seven patients there was a history of deep vein thrombosis; in four this occurred at the same time as the episode of subacute massive pulmonary embolism and in three had resolved at least six months beforehand.

\section{PHYSICAL SIGNS}

Dyspnoea at rest or on slight exertion was the most constant physical sign and was present in 18 of the 24 patients. Clinically detectable central cyanosis was present in eight patients.

Although none of the patients was clinically shocked and only one had a systolic blood pressure of less than $100 \mathrm{mmHg}$, many had physical signs that suggested that the cardiac output was compromised. Resting tachycardia was common; of the 24 patients, only eight had a resting heart rate of less than 100 , and in six patients the resting heart rate was between 120 and 140. Three patients had cool, cyanosed peripheries as well as resting tachycardia and one of these patients was the only patient with a blood pressure of less than $100 \mathrm{mmHg}(90 / 60 \mathrm{mmHg})$. The systolic blood pressure was between 100 and $110 \mathrm{mmHg}$ in five patients, between 110 and 120 $\mathrm{mmHg}$ in four, and above $120 \mathrm{mmHg}$ in the remainder.

The jugular venous pressure was raised more than $2 \mathrm{~cm}$ above the sternal angle at $45^{\circ}$ in 11 patients (range 3 to $10 \mathrm{~cm}$ ). In two of these patients, there was a dominant " $v$ " wave to a height of $10 \mathrm{~cm}$ above the sternal angle accompanied by a pansystolic murmur at the left sternal border. In both, the jugular venous pressure fell and the murmur disappeared as the patients recovered. On clinical grounds these physical signs were thought to represent functional tricuspid regurgitation; both patients had high pulmonary artery pressures at their initial study (70 $\mathrm{mmHg}$ systolic) when these signs were present. The cardiac impulse at the left sternal border was unusually prominent in four patients and 11 patients had a gallop rhythm detectable at this site. In general a raised jugular venous pressure, parasternal heave, or gallop rhythm did not predict a particularly raised pulmonary artery pressure, except in the two patients with tricuspid regurgitation.

In contrast, abnormalities of the second heart sound occurred only in the patients with the highest pulmonary artery pressures. The systolic pulmonary artery pressure exceeded $55 \mathrm{mmHg}$ in 10 patients and nine of these patients had abnormalities of the second heart sound; in five patients $P_{2}$ was accentuated (systolic pulmonary artery pressures 55, 61, 63, 
Table 1 Chest $\mathrm{x}$-ray and electrocardiogram at presentation

\begin{tabular}{|c|c|c|c|}
\hline & Abnormality & No. & of patients \\
\hline Chest $x$-ray & $\begin{array}{l}\text { Oligaemia } \\
\text { Consolidation or } \\
\text { collapse } \pm \text { pleural fluid } \\
\text { Enlarged main } \\
\text { pulmonary artery }\end{array}$ & $\left.\begin{array}{r}18 \\
20 \\
4\end{array}\right\}$ & in 22 patients \\
\hline Electrocardiogram & $\begin{array}{l}\text { Sinus tachycardia } \\
\text { Atrial flutter } \\
\quad(2: 1 \text { block }) \\
\mathrm{S}_{1} \mathrm{Q}_{3} \mathrm{~T}_{3} \\
\mathrm{~T} \text { inversion V } 1-3 \\
\text { Partial right bundle- } \\
\text { branch block }\end{array}$ & $\left.\begin{array}{r}16 \\
1 \\
12 \\
12 \\
2\end{array}\right\}$ & \}in 19 patients \\
\hline
\end{tabular}

75 , and $75 \mathrm{mmHg}$ ) and in four patients $P_{2}$ was of normal intensity but delayed, producing wide splitting of the second sound (systolic pulmonary artery pressures $55,70,70$, and $71 \mathrm{mmHg}$ ). An accentuated $\mathrm{P}_{2}$ was also heard in one of the two patients who were not catheterised.

In 12 of the 24 patients abnormal chest signs suggesting pleural fluids, pulmonary consolidation, or collapse or pleuritic inflammation were present. Fever occurred in 10 patients.

INITIAL CHEST $x$-RAY AND

ELECTROCARDIOGRAM

These results are summarised in Table 1. In all but two patients the chest $x$-ray was obviously abnormal. The electrocardiogram was completely normal in two patients and in three others the only abnormality was sinus tachycardia.

PULMONARY ANGIOGRAPHY AND

PULMONARY ARTERY PRESSURE

Right heart catheterisation and pulmonary angiography were performed in 22 patients.

The pulmonary angiographic score was $21 \cdot 4 \pm 3 \cdot 5$ (mean \pm SD), the systolic pulmonary artery pressure $56 \cdot 2 \pm 15 \cdot 7 \mathrm{mmHg}$ (mean $\pm \mathrm{SD}$ ), and the mean pulmonary artery pressure was $36 \cdot 7 \pm 9 \cdot 7 \mathrm{mmHg}$

Table 2 Treatment and outcome in subacute massive pulmonary embolism

\begin{tabular}{lclll}
\hline Initial treatment & \multirow{2}{*}{ No. } & \multicolumn{2}{l}{ Deaths } & \multirow{2}{*}{ Recovered } \\
\cline { 3 - 4 } & & Embolic & Other & \\
\hline Streptokinase & $14^{\star}$ & $4(1)$ & 1 & 9 \\
Heparin & 8 & $1(1)$ & 1 & $6(1)$ \\
Embolectomy & 2 & 2 & 0 & 0 \\
Total & 24 & 7 & 2 & 15
\end{tabular}

* Streptokinase changed to heparin at 24 hours because of haemoptysis in one patient.

Numbers in parentheses refer to the number of patients undergoing emergency embolectomy because of deterioration on their initial treatment. (mean $\pm \mathrm{SD})$. Both systolic and mean pulmonary artery pressure were significantly higher $(p<0.05)$ than in a group of 42 patients with acute massive pulmonary embolism ${ }^{13}$ with a similar degree of pulmonary artery obstruction. The angiographic score and pulmonary artery pressure did not differ between the patients with subacute massive pulmonary embolism who died during their initial illness and those who survived, nor between the different treatment groups.

\section{Clinical course and treatment during initial episode}

Treatment was with streptokinase, heparin, embolectomy, or a combination of these (Table 2). The treatment in the individual case was determined by the physician under whose care the patient was admitted. In general the more severely ill patients received streptokinase in the first instance, but since patients were not allocated randomly to different treatments it is difficult to compare their efficacy.

Nine patients $(38 \%)$ died during their initial illness. Seven of these deaths were directly attributable to embolic disease; the other two patients died of other serious concurrent illnesses (systemic lupus erythematosis and chronic lymphatic leukaemia) after showing signs of recovering from their embolic episode. Three of the seven patients who died of embolic disease did so during or after a 72 hour course of streptokinase; one deteriorated and died within 24 hours of starting treatment and the other two followed prolonged downhill courses, with a low cardiac output, during seven and 10 days, respectively, after starting treatment. The other four patients who died all underwent pulmonary embolectomy. In two the death was unrelated to embolectomy since one was moribund, receiving external cardiac massage having deteriorated on streptokinase treatment, and the other died of a massive recurrent pulmonary embolus despite adequate heparinisation several days after a successful embolectomy. In the other two patients, seen early in the series, embolectomy would no longer be considered the correct treatment. Neither patient was shocked and both would now be treated medically. One other patient underwent a successful embolectomy after deteriorating on heparin, making a total of five patients treated by embolectomy. Therefore of the seven patients, in whom embolic disease caused death, four deteriorated on medical treatment without evidence of a further embolus and in one of these embolectomy was attempted when the patient was moribund, one patient died of a further embolus after a successful embolectomy, and in two death 


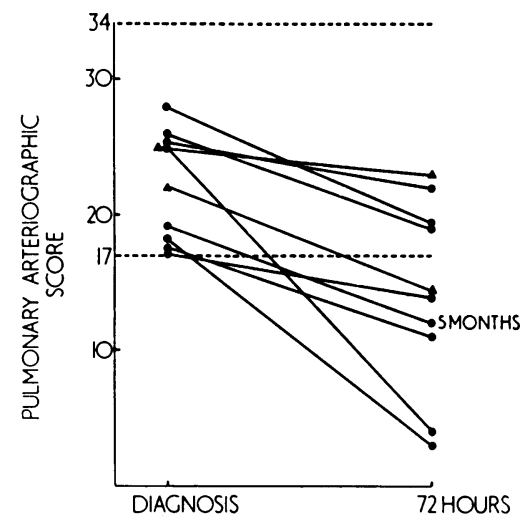

Fig. 1 The arteriographic response to initial treatment in subacute massive pulmonary embolism. The patients treated with streptokinase are represented by circles (O) and with heparin by triangles ( $\mathbf{\Delta})$.

was associated with pulmonary embolectomies, that in the light of present knowledge might have been avoidable.

Fig. 1 shows the early angiographic response to treatment in 10 patients. Nine of these patients were studied 72 hours after the beginning of treatment; the tenth patient was restudied at five months but was included with the early restudies, since the distribution of pulmonary angiographic abnormalities was the same as before treatment and there had been no clinical episode of recurrent embolism during the five month period. Only two patients showed dramatic clearing of the pulmonary arteries during the first 72 hours and both of these patients were receiving streptokinase. The early angiographic response to thrombolytic treatment was poor when compared with a group of 24 patients with acute massive pulmonary embolism receiving streptokinase (Fig. 2).

\section{Follow-up}

PATIENTS AVAILABLE FOR FOLLOW-UP

Of the 24 patients, 15 survived the initial illness. Two of these patients were lost to follow-up so that data were obtained in only 13 of the 15 survivors. Of these, one died two years after his initial illness of carcinoma of the pancreas, without clinical evidence of recurrent pulmonary embolism. The remaining 12 patients were recalled and seen five months to nine years (median five years) after their initial illness.

\section{INITIAL PREDISPOSING FACTORS}

Only four of the 13 patients followed up had welldefincd predisposing factors to embolic disease

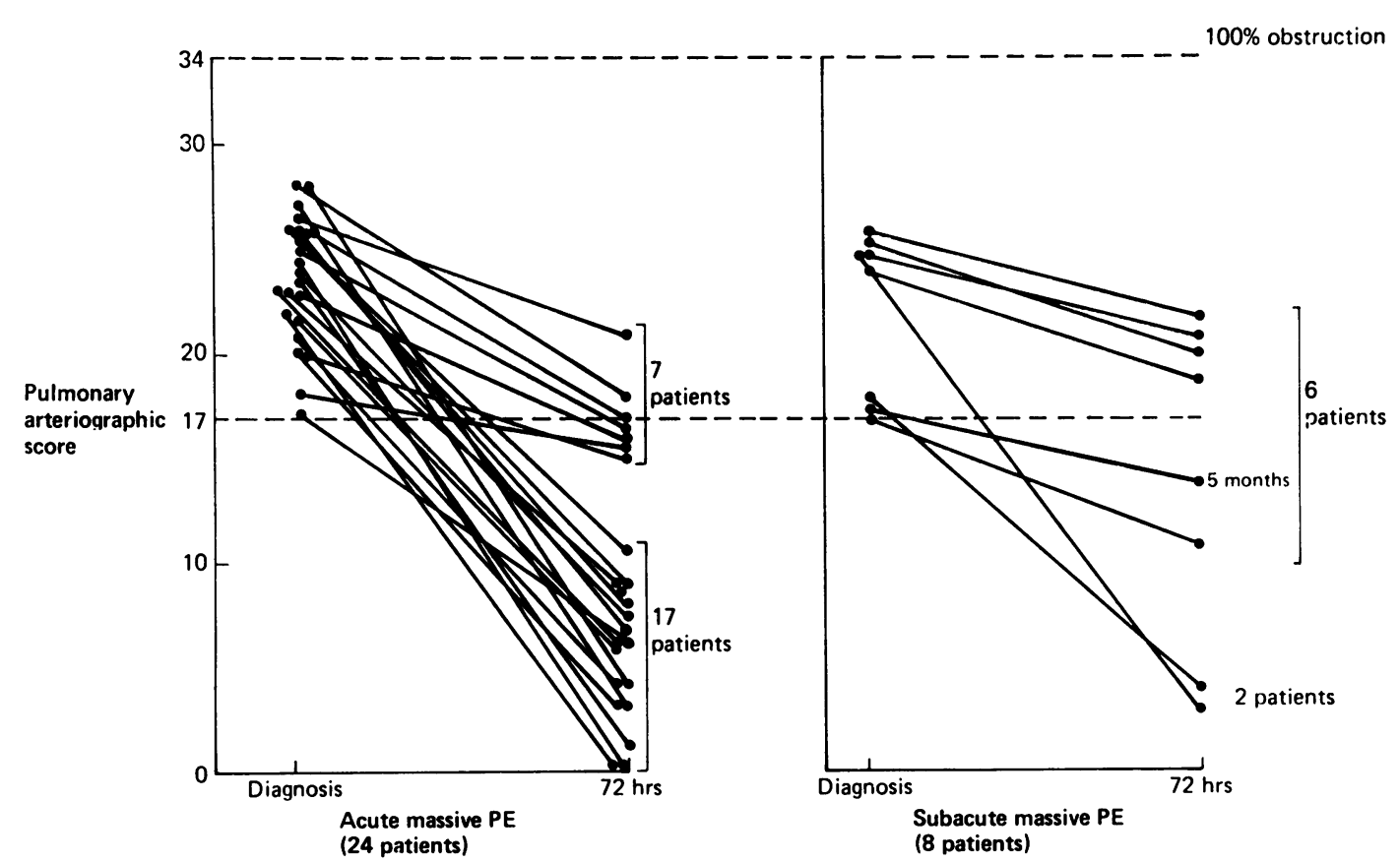

Fig. 2 Comparison of the arteriographic response to treatment with streptokinase in patients with acute and subacute massive pulmonary embolism. 
(the contraceptive pill-two patients, pregnancyone patient, severe obesity and primary lymphoedema-one patient). Three other patients gave long histories of recurrent phlebitis and had varicose veins.

\section{ANTICOAGULATION DURING FOLLOW-UP}

Seven patients received long-term, oral anticoagulants (warfarin) during the follow-up period and the remaining six patients were anticoagulated for two to 18 months after their initial illness. Venous interruption was not performed in any patient.

\section{CLINICAL FOLLOW-UP}

None of the 12 eventual survivors had had symptoms to suggest recurrent embolism and none had physical signs suggesting pulmonary hypertension. Ten of the 12 were asymptomatic (NYHA class 1 ). One grossly obese patient, who also suffered from primary lymphoedema, had severe exertional dyspnoea (NYHA class 3). She had no physical signs to suggest pulmonary hypertension or cardiopulmonary disease and repeat right heart catheterisation showed a normal resting pulmonary artery pressure $(25 / 12 \mathrm{mmHg})$ and mild, residual pulmonary angiographic abnormalities (score 8). It was concluded that her symptoms were largely caused by her gross obesity. The second patient with symptoms had mild exertional dyspnoea (NYHA class 2), a normal pulmonary artery pressure, and a completely normal pulmonary angiogram. Clinically he had chronic bronchitis which was almost certainly the cause of his symptoms. Therefore, in neither could non-resolution or recurrent pulmonary embolism be incriminated as the cause of disability.

\section{ELECTROCARDIOGRAM, CHEST $x$-RAY, AND LUNG SCAN}

Electrocardiograms and chest $x$-ray films were obtained in all 12 patients recalled. The electrocardiogram had returned to normal in 10 patients. Two patients had an abnormal electrocardiogram at follow-up. In one, the $S_{1} Q_{3} T_{3}$ pattern, partial right bundle-branch block, and $\mathrm{T}$ wave inversion in V2 to 6 seen during the initial illness was still present seven years later. This was the patient already described with severe dyspnoea, obesity, primary lymphoedema, and a normal pulmonary artery pressure. In the other patient $T$ wave inversion in V2 to 4 was still present one year later but the $\mathrm{S}_{1} \mathrm{Q}_{3} \mathrm{~T}_{3}$ pattern, initially present, had resolved. This patient had a normal pulmonary artery pressure and only minor pulmonary angiographic abnormalities at follow-up. The chest $x$-ray film was normal in nine patients. In two, there were some areas of oligaemia and both patients showed some residual pulmonary angiographic abnormalities (scores of 7 and 8). One patient who still had severe residual abnormalities at repeat pulmonary angiography (case 1), had a grossly abnormal chest $x$-ray with a raised diaphragm and considerable shadowing in the left lung, the one in which nearly all the persistent angiographic abnormalities were seen.

Late lung scans were carried out in eight of the 12 patients. These were normal in three. Three others showed multiple perfusion defects and two had single defects. All five patients with abnormal lung scans showed minor angiographic abnormalities but in the three patients with multiple perfusion defects, the extent of the abnormalities on lung scan greatly exceeded the extent of those on angiography.

\section{CARDIAC CATHETERISATION AND PULMONARY ANGIOGRAPHY}

Nine of the 12 patients recalled had a right heart catheterisation and pulmonary angiography (Table 3). Two other patients who had complete angiographic and haemodynamic resolution at a restudy 72 hours after the onset of initial treatment and no subsequent symptoms were not restudied but their results are included with the late restudy data. One patient who had not had an early restudy but had been completely well for the subsequent three years refused investigation. One patient had a significantly raised pulmonary artery pressure but this was the result of left ventricular dysfunction with a pulmonary artery wedge pressure of 20 $\mathrm{mmHg}$ and the pulmonary angiogram and pulmonary vascular resistance were normal. Three other patients (cases 1, 8, and 9) had mildly raised ( $>30 \mathrm{mmHg}$ ) systolic pulmonary artery pressures and in these patients some residual pulmonary angiographic abnormalities were present. Five other patients showed persistent angiographic abnormalities. In four these were minor consisting of mildly reduced perfusion and loss of a few small peripheral pulmonary arteries. In one patient, restudied at five months, considerable pulmonary artery obstruction persisted though the systolic pulmonary artery pressure was almost normal (33 $\mathrm{mmHg}$ ) and the patient was asymptomatic.

\section{Discussion}

(A) DIAGNOSIS AND TREATMENT OF INITIAL ILLNESS

Subacute massive pulmonary embolism is a wellrecognised but rare condition. It is frequently referred to as "recurrent pulmonary embolism" 
Table 3 Systolic pulmonary artery pressure and pulmonary angiographic score at diagnosis and restudy in 12 survivors of subacute massive pulmonary embolism

\begin{tabular}{|c|c|c|c|c|c|c|c|c|c|}
\hline \multirow{2}{*}{$\begin{array}{l}\text { Case } \\
\text { No. }\end{array}$} & \multirow[t]{2}{*}{ Age, sex } & \multicolumn{3}{|l|}{ First study } & \multicolumn{5}{|c|}{ Second study } \\
\hline & & $\begin{array}{l}\text { Initial } \\
\text { treatment }\end{array}$ & $\begin{array}{l}\text { Systolic } P A \\
\text { pressure } \\
(m m H g)\end{array}$ & $\begin{array}{l}\text { Pulmonary } \\
\text { angio score }\end{array}$ & $\begin{array}{l}\text { Interval } \\
\text { from 1st } \\
\text { study }\end{array}$ & $\begin{array}{l}\text { Systolic } P A \\
\text { pressure } \\
(\mathrm{mmHg})\end{array}$ & $\begin{array}{l}\text { Pulmonary } \\
\text { angio score }\end{array}$ & $\begin{array}{l}\text { Symptoms } \\
\text { at follow-up }\end{array}$ & Comment \\
\hline 1 & $20 \mathrm{~F}$ & SK & 33 & 17 & $5 \mathrm{mth}$ & 33 & 14 & 0 & \\
\hline 2 & $59 \mathrm{~F}$ & SK & 75 & $26(22)^{\star}$ & $7 y$ & 25 & 8 & ++ & Gross obesity \\
\hline 3 & $45 \mathrm{M}$ & SK & 70 & $25(4)^{\star}$ & $7 \mathrm{y}$ & 28 & 0 & 0 & \\
\hline 4 & $45 \mathrm{M}$ & Hep & 45 & $22(16)^{\star}$ & $9 \mathrm{y}$ & 22 & 0 & 0 & \\
\hline 5 & $48 \mathrm{~F}$ & Hep & 60 & 19 & $4 \mathrm{y}$ & 43 & 0 & 0 & $\begin{array}{l}\text { Normal PVR } \\
\text { LV disease }\end{array}$ \\
\hline 6 & $31 \mathrm{M}$ & Hep & 50 & 18 & - & - & - & - & Refused study \\
\hline 7 & $38 \mathrm{~F}$ & SK & 60 & $26(19)^{\star}$ & $5 y$ & 24 & 0 & 0 & \\
\hline 8 & $71 \mathrm{M}$ & Hep & 71 & 22 & $3 \mathrm{mth}$ & 33 & 7 & 0 & \\
\hline 9 & $64 \mathrm{M}$ & SK & 60 & 21 & $1 \mathrm{y}$ & 37 & 6 & 0 & \\
\hline 10 & $35 \mathrm{~F}$ & SK & 55 & $25(23)^{\star}$ & $5 \mathrm{mth}$ & 30 & 4 & 0 & \\
\hline 11 & $55 \mathrm{M}$ & Hep + embol & 40 & $18(0)^{\star}$ & $3 d$ & 27 & 0 & + & $\begin{array}{l}\text { Chronic } \\
\text { bronchitis }\end{array}$ \\
\hline 12 & $48 \mathrm{~F}$ & SK & 35 & $18(3)$ & $3 \mathrm{~d}$ & 30 & 3 & 0 & \\
\hline
\end{tabular}

SK, streptokinase; Hep, heparin; embol, embolectomy; PVR, pulmonary vascular resistance.

*Pulmonary angio score after three days of treatment.

but this description does not distinguish those patients who have repeated pulmonary emboli well separated in time from this group of patients in whom the result is a steadily progressive illness. Though repeated episodes of embolism may be recognised early if typical features such as pleuritic pain and haemoptysis are prominent, these patients often present with advanced pulmonary artery obstruction. There are two main reasons for this. Firstly since recognisable predisposing factors for thromboembolic disease are frequently absent these episodes are often misdiagnosed as chest infections. ${ }^{4}$ Secondly, when the history is, as so often, of increasing dyspnoea alone and the physical signs are inconspicuous the illness may not be recognised until at a late stage severe symptoms demand further assessment and investigation.

Occasionally the patient presents with very few helpful physical signs and with a normal chest $x$-ray film and electrocardiogram. In this unusual situation the most helpful clue to the true diagnosis is the history of progressive dyspnoea over a period of a few weeks. In the majority of patients, however, the physical signs combined with the chest $x$-ray and electrocardiographic findings point towards the true diagnosis. Dyspnoea at rest or on slight exertion, without evidence of obvious left heart failure, airways obstruction, or chronic lung disease, is the most prominent feature. A history of episodes that could represent pulmonary infarction, and a pronounced sudden increase in symptoms on the background of steady deterioration is also helpful though often not present.

Many patients also have sinus tachycardia, cyanosis at rest or on mild exertion, a raised jugular venous pressure, or a gallop rhythm at the left sternal border to help in diagnosis. More rarely, evidence of pulmonary hypertension in the form of an accentuated pulmonary component of the second heart sound may occur. This was found in the five of our patients with the highest pulmonary artery pressures. Even more infrequently there may be tricuspid regurgitation that resolves with successful treatment. Neither of these physical signs is a feature of acute massive pulmonary embolism.

Since these patients usually present late, and are often not under medical observation during the early stages of the illness, angiographic evidence for the way that the pulmonary artery obstruction occurs is not available, nor is there any explanation in many patients why embolic disease should occur. Despite this, it is a reasonable assumption that it is caused by the accumulation of multiple small and medium sized emboli in the pulmonary vascular bed over a period of weeks. The occurrence of discrete episodes of pleuritic pain and haemoptysis in some patients, the progressive increase in symptoms, the occasional sudden exacerbation of symptoms possibly caused by a larger embolus, and the finding of clot of different ages at embolectomy or necropsy all suggest that this is the mechanism. Such a progression would explain the observed differences in pulmonary artery pressure in patients with subacute and acute massive pulmonary embolism with the same degree of pulmonary artery obstruction. In acute massive pulmonary embolism the pulmonary vascular bed is suddenly obstructed and the acutely stressed, thin-walled right ventricle is unable to generate a systolic pulmonary artery pressure much in excess of 50 $\mathrm{mmHg}$. In contrast, in subacute massive pulmonary 
embolism, the right ventricle has had time to adapt and hypertrophy as the pulmonary vascular resistance slowly increases and therefore can generate much higher pulmonary artery pressures. The clinical consequence of this haemodynamic difference is that, on occasion, signs of pulmonary hypertension are a feature of subacute massive pulmonary embolism but they never occur in acute massive pulmonary embolism.

The electrocardiogram and chest $x$-ray film were useful clues to the correct diagnosis. Nearly 80 per cent ( 19 of 24 patients) had electrocardiographic changes suggesting right heart strain. None had evidence of right ventricular hypertrophy, but this does not mean that this did not develop during the course of the illness since the electrocardiogram is notoriously insensitive to mild degrees of right ventricular hypertrophy. ${ }^{5}$ Similarly, the chest $x$-ray film was extremely helpful. Twenty-two of the 24 patients had either obvious oligaemic zones or, more frequently, $x$-ray changes consistent with pulmonary infarction. We do not have lung scans during the initial illness in these patients, but perfusion lung scans are likely to be grossly abnormal in most patients with this condition and a normal perfusion scan should rule out the diagnosis.

Analysis of the resuits of treatment is difficult since individual patients were treated according to the wishes of the physician under whom they were admitted. In general the more severely ill patients received streptokinase and this probably accounts for the larger number of failures with this treatment when compared with heparin. Comparison of the effect of streptokinase in subacute and acute massive pulmonary embolism suggests that the rapid clearing of the pulmonary circulation that occurs so frequently in the latter is uncommon in the former. This is not surprising since much of the pulmonary artery obstruction may be caused by clot that is too old to be lysed by streptokinase. This explanation is borne out by our limited experience of pulmonary embolectomy in these patients, when despite angiographically demonstrated massive proximal pulmonary artery obstruction all that could be obtained at operation were small amounts of the more proximal thrombus, friable and often adherent to the pulmonary arteries. This contrasts with the situation in acute massive pulmonary embolism when the surgeon is usually able to pull out several hundred grams of clot. These differences in the surgical findings also explain the generally dismal results of embolectomy.

Since heparin appeared as effective as streptokinase in the majority of patients we suggest that all patients with subacute massive pulmonary embolism should receive intravenous heparin as initial treatment. If deterioration continues despite heparin thrombolytic treatment should be used since it is spectacularly successful in a few patients. If this fails embolectomy should be undertaken as a last resort. All patients should receive full intravenous heparinisation until they are fully mobile and only then be transferred to oral anticoagulants.

\section{(B) LATE PROGNOSIS}

Treated acute massive pulmonary embolism has a good long-term prognosis, and recurrent pulmonary embolism, poor resolution of pulmonary artery obstruction, and the development of pulmonary hypertension are extremely rare. ${ }^{2} 6$ This is not surprising since most of these patients have welldefined and often temporary factors predisposing to embolism, the embolus is of recent formation, and it is susceptible to both therapeutic and natural lysis. In contrast, in subacute massive pulmonary embolism the predisposing factor is often unknown and potentially might continue to operate after initial treatment, causing recurrence of emboli. Furthermore, older clot, accumulated in the pulmonary circulation over a period of weeks, might be expected to lyse less easily. If so, thromboembolic pulmonary hypertension ought to be more likely to develop in subacute rather than in acute massive pulmonary embolism. This, however, was not our experience. Recurrent pulmonary embolism did not occur. Since several patients received long-term anticoagulants these could have been important but there is insufficient information to assess this. The lack of recurrence cannot be attributed to surgical interruption of venous drainage since no such operations were performed, and our results suggest that such treatment is not indicated in patients with a single episode of subacute massive pulmonary embolism. Resolution of pulmonary artery obstruction assessed angiographically was generally good though minor abnormalities persisted in some patients and one patient showed significant abnormalities without pulmonary hypertension or symptoms when restudied only five months after the initial episode. The rather more frequent and widespread perfusion abnormalities, seen on perfusion lung scans, are similar to the findings in acute massive pulmonary embolism $^{2}$ and suggest that long-term damage to vessels smaller than those seen angiographically is common. The patients we describe must be distinguished from those with repeated, discrete episodes of embolism, well separated in time, who are even less commonly seen than patients with subacute massive pulmonary embolism, and appear to have a poor prognosis. ${ }^{7}$ Though there is no 
controlled evidence for the benefits of long-term anticoagulants in subacute massive pulmonary embolism, our policy in this group of patients has been to give oral anticoagulants indefinitely unless a well-defined, temporary predisposing factor for the episode was identified or there was a strong contraindication to this treatment.

Therefore, patients surviving an episode of subacute massive pulmonary embolism have a good long-term prognosis and recurrent embolism is rare. Resolution of pulmonary artery obstruction is usually good though minor abnormalities of the pulmonary vasculature may persist which are not associated with significant pulmonary hypertension of clinical disability.

\section{References}

1 Sutton GC, Hall RJC, Kerr IH. Clinical course and late prognosis of treated subacute massive, acute minor, and chronic pulmonary thromboembolism. Br Heart f 1977; 39: 1135-42.

2 Hall RJC, Sutton GC, Kerr IH. Long-term prognosis of treated acute massive pulmonary embolism. Br Heart f 1977; 39: 1128-34.

3 Miller GAH, Sutton GC, Kerr IH, Gibson RV, Honey M. Comparison of streptokinase and heparin in treatment of isolated acute massive pulmonary embolism. Br Med f 1971; ii: 681-4.

4 Fleischner FG. Recurrent pulmonary embolism and cor pulmonale. $N$ Engl f Med 1967; 276: 1213-20.

5 Flowers NC, Horan LG. Subtle signs of right ventricular enlargement and their relative importance. In: Schlant RC, Hurst JW, eds. Advances in electrocardiography. New York: Grune \& Stratton, 1972: 297-308.

6 Paraskos JA, Adelstein SJ, Smith RE, et al. Late prognosis of acute pulmonary embolism. $N$ Engl f Med 1973; 289: 55-8.

7 Riedel M, Prerovsky I, Stanek V, Ressl J, Stadlerova V, Widimsky J. Chronic thromboembolic disease. Long-term follow-up. Prog Respir Res; 13: 134-40.

Requests for reprints to Dr R J C Hall, The Royal Victoria Infirmary, Queen Victoria Road, Newcastle Upon Tyne NE1 4LP. 